\title{
Periodic Solutions and Asymptotic Analysis of Ordinary Differential Equations
}

\author{
Maoan Han, ${ }^{1}$ Pei Yu, ${ }^{2}$ Valery G. Romanovski, ${ }^{3}$ and Tonghua Zhang ${ }^{4}$ \\ ${ }^{1}$ Department of Mathematics, Shanghai Normal University, Shanghai 200234, China \\ ${ }^{2}$ Department of Applied Mathematics, University of Western Ontario, London, ON, Canada N6A 5B7 \\ ${ }^{3}$ Center for Applied Mathematics and Theoretical Physics and Faculty of Natural Science, University of Maribor, Krekova 2, \\ Maribor 2000, Slovenia \\ ${ }^{4}$ Department of Mathematics, Swinburne University of Technology, Melbourne Vic 3122, Australia
}

Correspondence should be addressed to Maoan Han; mahan@shnu.edu.cn

Received 15 December 2013; Accepted 15 December 2013; Published 13 February 2014

Copyright (c) 2014 Maoan Han et al. This is an open access article distributed under the Creative Commons Attribution License, which permits unrestricted use, distribution, and reproduction in any medium, provided the original work is properly cited.

Ordinary Differential Equations (ODEs) arise in many different aspects throughout mathematics and science (social and natural) one way or another and have a very wide range of applications in many fields. In recent years, many researchers have done a lot of studies in this field and made significant advances both in theory and in applications.

With the intention to provide an opportunity for the researchers in the area to report their most recent research findings, we proposed this special issue. It mainly focuses on periodic solutions and asymptotic analysis of ODEs, exploring the latest advances in the theory and in applications of ODEs. We have received numerous contributions, which cover very wide range of topics such as bifurcation theory and its application, stochastic systems, theory of limit cycles, and asymptotic analysis of systems with time delay. All papers submitted to this special issue had been sent to at least three external reviewers for peer review. Based on the reviewers' reports, we carefully selected some original works for publication.

In a single special issue, of course, there is no way to cover all recent advances in the area of Ordinary Differential Equations. But we do believe that the results published in this issue at least can reflect some of the most current trends in the area of Ordinary Differential Equations.

\section{Acknowledgments}

The guest editors of this special issue would like to take this opportunity to thank all our contributors for submitting their excellent work to this issue. Also, we extend our thanks to all the reviewers for being happy to be reviewers and their hard work. Finally, we extend our thanks to the editorial board members of this journal for their technical support and help during the whole period.

Maoan Han

$\mathrm{Pei} \mathrm{Yu}$

Valery Romanovski

Tonghua Zhang 


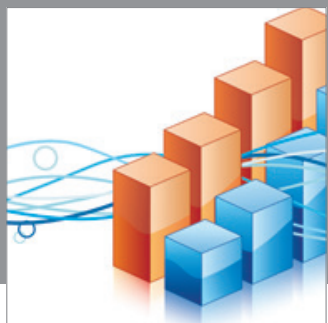

Advances in

Operations Research

mansans

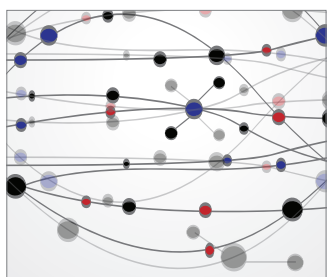

The Scientific World Journal
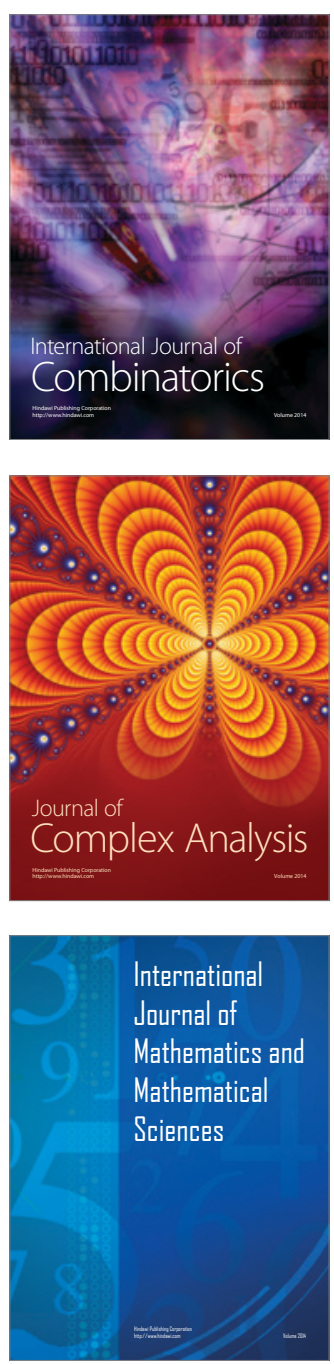
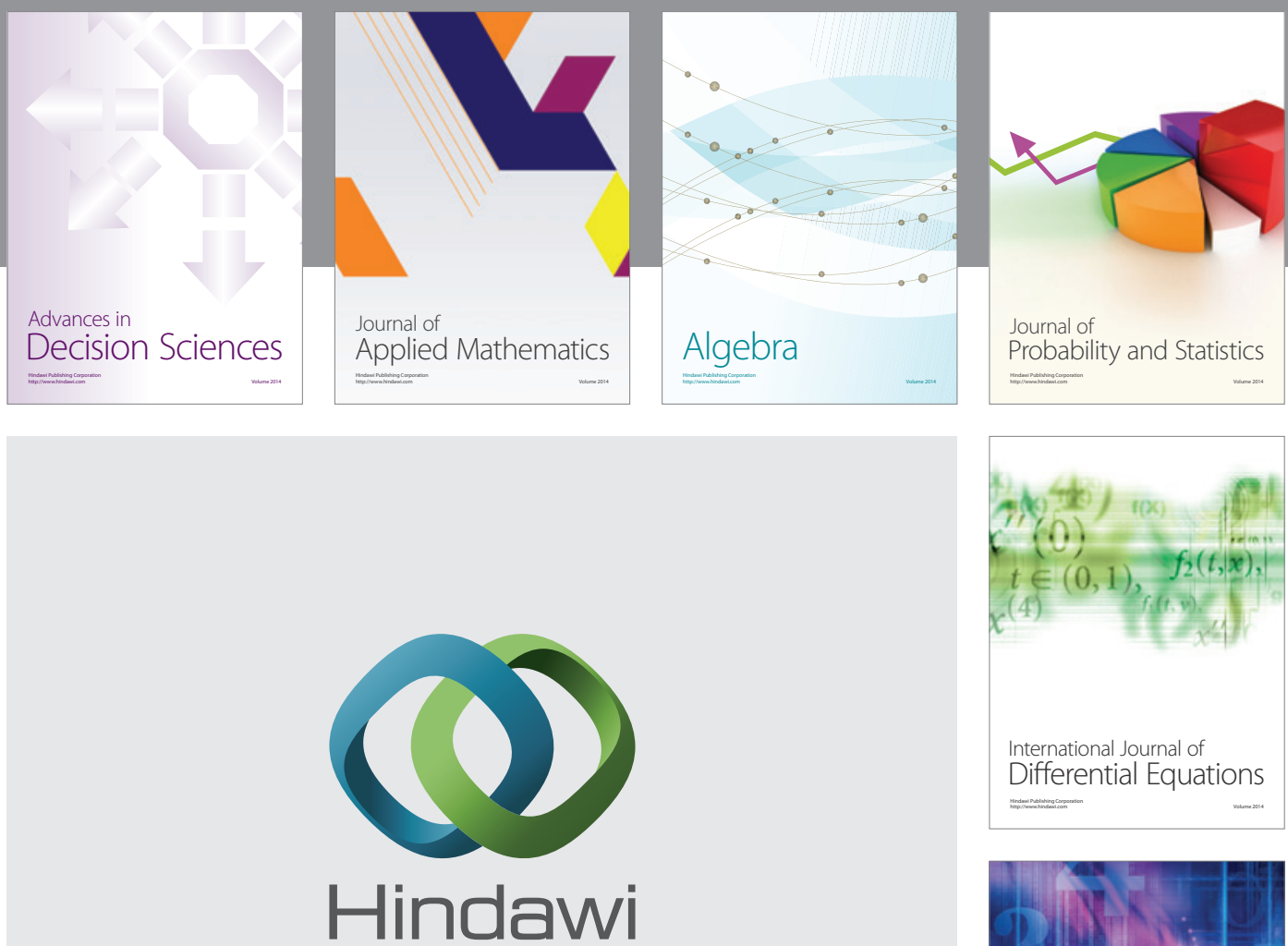

Submit your manuscripts at http://www.hindawi.com
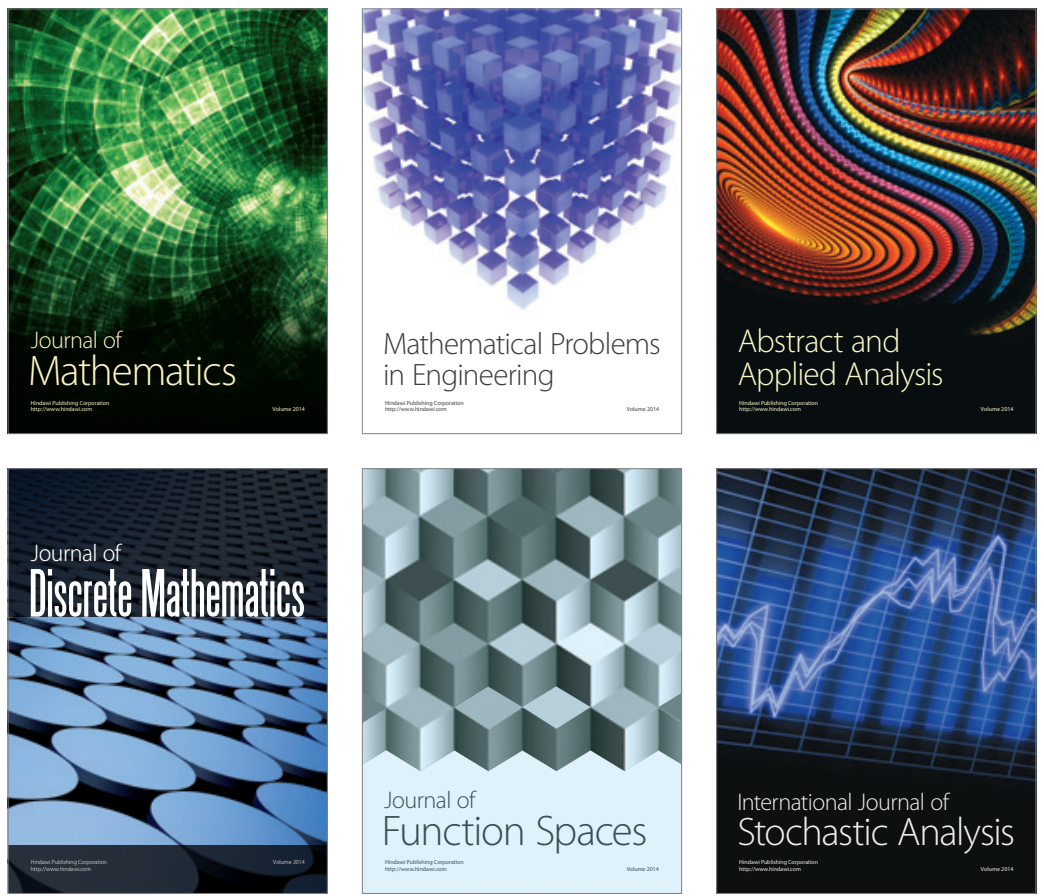

Journal of

Function Spaces

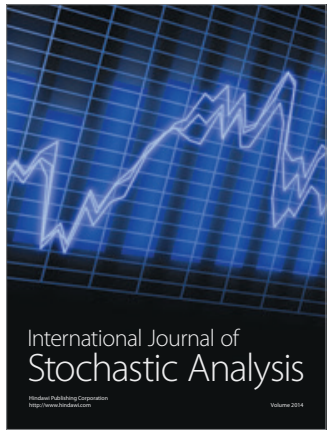

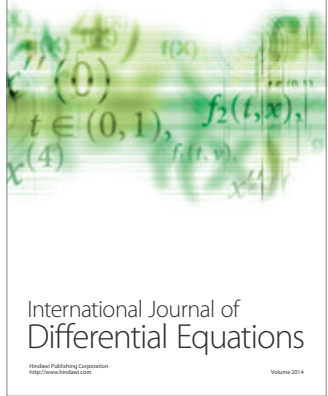
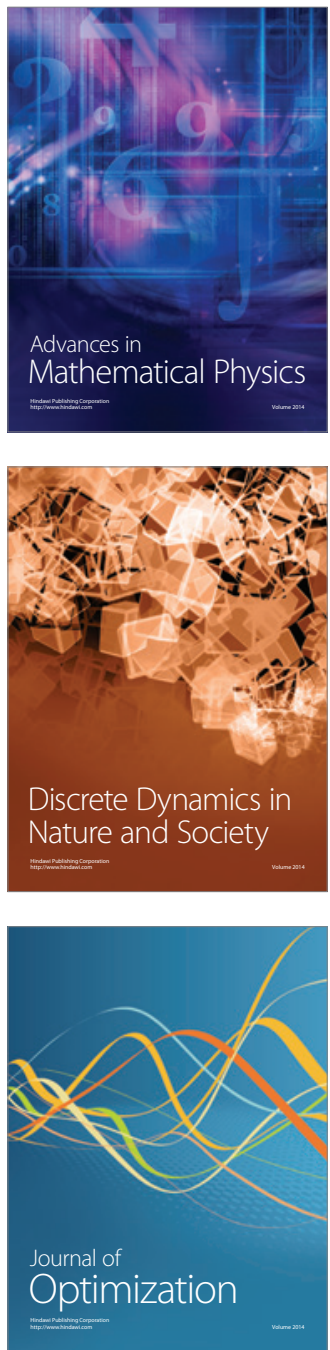